\title{
Capacity Analysis of an AF Relay Cooperative NOMA System Using MRC
}

\author{
Xianbin $\mathrm{Xie}^{{ }^{*}}$, Yan $\mathrm{Bi}^{2}$ and $\mathrm{Xi} \mathrm{Nie}{ }^{1}$ \\ ${ }^{1}$ Department of Big Data Engineering, Guizhou Institute of Technology \\ Guiyang, China \\ [e-mail: xianbinxie_uestc@163.com; niexixy@163.com] \\ ${ }^{2}$ Department of Mathematics and Physics, Guizhou Institute of Technology \\ Guiyang, China \\ [e-mail: biyan1015@163.com] \\ *Corresponding author: Xianbin Xie
}

Received June 10, 2020; revised September 5, 2020; accepted September 22, 2020; published October 31, 2020

\begin{abstract}
Non-orthogonal multiple access (NOMA) is widely studied in both academia and industry due to its high spectral efficiency over orthogonal multiple access (OMA). To effectively improve spectrum efficiency, an amplify-and-forward (AF) cooperative NOMA system is proposed as well as a novel detection scheme is proposed, in which we first perform successive interference cancellation (SIC) twice at U1 for the two signals received from two time slots to remove interference from symbol 2, then two new signals apply max ratio combining (MRC). In addition, a closed-form upper bound approximation for the ergodic capacity of our proposed system is derived. Monte-Carlo simulations and numerical analysis illustrate that our proposed system has better ergodic capacity performance than the conventional cooperative NOMA system with decode-forward (DF) relay, the conventional cooperative NOMA system with AF relay and the proposed AF cooperative NOMA system in [16]. In addition, we can see that ergodic capacity of all NOMA cooperative systems increase with the increase of transmit SNR. Finally, simulations display that power allocation coefficients have little effect on ergodic capacity of all NOMA cooperative systems. This is due to this fact that ergodic capacity of two symbols can be complementary with changing of power allocation coefficients.
\end{abstract}

Keywords: NOMA, Max ratio combining, amplify-and-forward, decode-and-forward, ergodic capacity

This work has been supported by the Joint Foundation of Guizhou Province (QianKeHeLHZi[2016]7106) , XJGC20150702 and the Nature Science Foundation of Guizhou Province (QianKeHeJiChu[2017]1070). 


\section{Introduction}

As a effective solution to obtain higher transmission rate, the non-orthogonal multiple access (NOMA) has attracted tremendous interests in both industry and academia [1]. The main idea of NOMA is to adopt the superposition coding at the transmitting terminal as well as utilize the successive interference cancellation (SIC) at the receiving terminal [2]. Especially recently, some novel research works have appeared on NOMA [3-5]. A new definition of fairness for uplink NOMA was proposed based on power domain by Gui et al. [3]. To effectively improve energy efficiency (EE) and spectrum efficiency (SE). Wang et al. [4] proposed a novel technology, which multiple unmanned aerial vehicles (UAVs) aided NOMA technology to enhance the EE and the SE of the uplink cellular systems. In addition, Gui et al. [5] look ahead a new development trend of NOMA technology in the future sixth generation (6G) wireless networks.

Cooperative communication is an effective scheme to overcome multipath propagation as well as enhance reception reliability of communication systems. Interplay between cooperative communication and NOMA constitute the cooperative NOMA systems which is widely studied in recent years $[6-14,16]$. According to the difference of selected relay node, the cooperative NOMA systems are mainly divided into two types: The first type of cooperative NOMA systems utilizes one NOMA user as the relay, while, the second type of cooperative NOMA systems mainly uses the dedicated relay as the relay.

There are many research works have been undertaken for the first type of cooperative NOMA systems. Ding and co-authors [6] proposed a cooperative NOMA system, where the user of strong channel gain as the relay forwards the decoded messages to the user of poor channel. Compared with the conventional NOMA system and the conventional cooperative OMA system, the proposed cooperative NOMA system obtains higher ergodic capacity as well as better fairness. Xu et al. [7] proposed using maximum ratio combining ( MRC) to improve the gain of spatial diversity for cooperative NOMA system. In addition, Ding et al. [8] proposed a two-phase relay selection operating mode to reduce the outage probability of the proposed cooperative NOMA system. To further make better the performance of system, the Full-duplex (FD) work mode also was applied to cooperative NOMA system [9-11].

Based on the second type of cooperative NOMA systems, the main works are as follows: Men et al. [12] analyzed the outage performance of the proposed cooperative NOMA system, where the system adopted a multi-antenna as the relay with AF transmission protocol. In addition, a novel two-way relay selection cooperative NOMA system was also proposed by Yue et al. [13]. Furthermore, considering the uplink situation, a uplink cooperative NOMA system was proposed with relay-aided for multi-cell circumstances [14].

The aforementioned works based on the second type of cooperative NOMA systems assume that dose not exist direct links between the base station (the source) and the downlink users (the destination). Namely, all users obtain information need through the dedicated relay forward information. However, in some typical small cells 5G scenarios [15], part users can directly communicates with the base station, while others can not directly communicates with the base station. Actually, based on the second type of cooperative NOMA scheme considering both direct and indirect links users is also addressed. In particular, a recent work addressed in [16], a cooperative NOMA system was proposed with an amplify-and-forward (AF) relay as well as considering the direct link user, where the system outage performance was analyzed. However, the ergodic capacity based on [16] system mode has not yet been 
investigated, which inspired the investigation of this paper. In addtion, the difference between our work and [16] also lies in the signal detection method. To eliminate interference from symbol 2, we first perform SIC twice at the user U1 to two signals received from two time slots in our proposed system, then we apply MRC to two new signals. Compared with [16], which first applies MRC next applies SIC, our proposed detection scheme obtains more received diversity gain for the symbol 1 .

The preliminary results given in this paper including:

- Giving an AF relay cooperative NOMA system and a novel detection scheme is proposed, in which we first perform SIC two times for two signals received from two continuous time slots to remove interference from symbol 2. Then, using two signals after being eliminated symbol 2 by perform SIC to apply MRC.

- A closed-form upper bound approximation analytical expression for ergodic capacity of our proposed system is derived. In this procedure, we also provide four lemmas and five propositions.

- Simulations show that our proposed system has higher ergodic capacity than the conventional cooperative NOMA system with AF/DF relay and [16] proposed cooperative NOMA system.

The structure of this paper is as follows:

Section 2 gives the system model. Next, ergodic capacity performance is analyzed in Section 3. The numerical results are discussed in Section 4. Finally, the Section 5 is the conclusion.

\section{System Model}

We consider a downlink cooperative NOMA system including one base station (BS), two users(U1 and U2) and one relay in Fig. 1. We assume that U1 is closer to the base station than U2. Hence, U1 can directly communicate with the BS without needing help of relays. The BS and U2 does not exist a direct link because of the long distance or heavy shadowing, thus, communicates between the BS and U2 always need help of the relay. We also assume that, each node is equipped with a single-antenna and operates in half-duplex (HD) mode. In addition, the relay adopts an AF transmission protocol. Subscript S, R, 1 and 2 represents the BS (the source), the relay, U1, and U2. The channel coefficients for the link from the BS to the relay, the link from the BS to U1, the link from the relay to U1, and the link from the relay to U2 are denoted by $h_{S R}, h_{S 1}, h_{R 1}$, and $h_{R 2}$, respectively. Here, all channels are assumed for Rayleigh fading channels. Therefore, all channel power gain obey exponential distributed. The mean of the channel power gains $\left|h_{S R}\right|^{2},\left|h_{S 1}\right|^{2},\left|h_{R 1}\right|^{2}$, and $\left|h_{R 2}\right|^{2}$ are defined as $\lambda_{S R}^{\prime}, \lambda_{S 1}^{\prime}$, $\lambda_{R 1}^{\prime}$, and $\lambda_{R 2}^{\prime}$, respectively.

For the convenience of the following description, we assume that the time slot $n$ represents the first slot time as well as the time slot $n+1$ represents the second time slot . At the first time slot, the BS broadcasts the superimposed signal $x[n]=\sqrt{P \alpha_{1}} x_{1}[n]+\sqrt{P \alpha_{2}} x_{2}[n]$, where $P$ denotes the transmission power at the BS. Without loss of generality, we also assume that the relay transmission power is the same as the BS transmission power. Let $x_{1}[n]$ and $x_{2}[n]$ denotes symbol 1 for $\mathrm{U} 1$ and symbol 2 for $\mathrm{U} 2$, respectively. $\alpha_{1}$ and $\alpha_{2}$ are power allocation coefficients, where satisfy $\alpha_{2}>\alpha_{1}$ and $\alpha_{1}+\alpha_{2}=1$. 


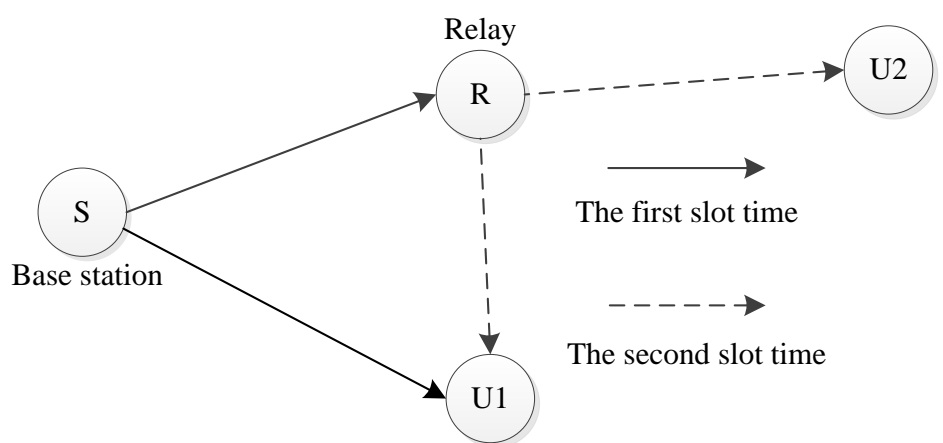

Fig. 1. System model

During the first time slot, the received signal $y_{R}[n]$ at the relay, and $y_{1}[n]$ at U1 are given by

$$
\begin{gathered}
y_{R}[n]=h_{S R}[n]\left(\sqrt{P \alpha_{1}} x_{1}[n]+\sqrt{P \alpha_{2}} x_{2}[n]\right)+N_{R}[n], \\
y_{1}[n]=h_{S 1}[n]\left(\sqrt{P \alpha_{1}} x_{1}[n]+\sqrt{P \alpha_{2}} x_{2}[n]\right)+N_{1}[n],
\end{gathered}
$$

where $N_{R}[n]$ and $N_{1}[n]$ denote the independent additive white Gaussian noises (AWGN) at the relay and U1, respectively, which satisfy normal distribution with zero mean and variance $\sigma^{2}=1$.

At the second time slot, according to AF protocol in cooperative communications, the relay broadcasts $\rho y_{R}[n]$ to $\mathrm{U} 1$ and $\mathrm{U} 2$ by an amplifying gain $\rho=\sqrt{\frac{P}{P\left|h_{S R}[n]\right|^{2}+\sigma^{2}}}$. Thus, the received signal at $\mathrm{U} 1$ and $\mathrm{U} 2$ are respectively given by

$$
\begin{aligned}
& y_{1}[n+1]=h_{R 1}[n+1] \rho y_{R}[n]+N_{1}[n+1], \\
& y_{2}[n+1]=h_{R 2}[n+1] \rho y_{R}[n]+N_{2}[n+1],
\end{aligned}
$$

where $N_{1}[n+1]$ and $N_{2}[n+1]$ also denote the independent AWGN, and also satisfy normal distribution with zero mean as well as variance $\sigma^{2}=1$, at $\mathrm{U} 1$ and $\mathrm{U} 2$, respectively.

For the convenience of the description, denotes $\gamma=\frac{P|h|^{2}}{\sigma^{2}}$, e.g. $\gamma_{S R}=\frac{P\left|h_{S R}\right|^{2}}{\sigma^{2}}$. Also, by defining $E[\gamma]=\lambda$, where $E[\cdot]$ represents statistical expectation. Hence, there is $\lambda=\frac{P}{\sigma^{2}} \lambda^{\prime}$, where variable $\gamma$ is also exponential distributed, the mean of the variables $\gamma_{S R}, \gamma_{S 1}, \gamma_{R 1}$, and $\gamma_{R 2}$ can be defined as $\lambda_{S R}, \lambda_{S 1}, \lambda_{R 1}$, and $\lambda_{R 2}$, respectively. To eliminate symbol 2 interference, we perform SIC twice at $\mathrm{U} 1$ to both of received signals $y_{1}[n]$ and $y_{1}[n+1]$. Here, assuming the SIC is perfect. Thus, the corresponding two new signals, in which generated after perform SIC, can be written as

$$
\begin{gathered}
y_{1}^{\prime}[n]=h_{S 1}[n] \sqrt{P \alpha_{1}} x_{1}[n]+N_{1}[n], \\
y_{1}^{\prime}[n+1]=h_{R 1}[n+1] \rho h_{S R}[n] \sqrt{P \alpha_{1}} x_{1}[n]+h_{R 1}[n+1] \rho N_{R}[n]+N_{1}[n+1] .
\end{gathered}
$$

Next, applying MRC for $y_{1}^{\prime}[n]$ and $y_{1}^{\prime}[n+1]$ at U1. Hence, the merged signal at U1 for decoding symbol 1 is given by 


$$
y_{1}=w_{1} y_{1}^{\prime}[n]+w_{2} y_{1}^{\prime}[n+1],
$$

where $w_{1}$ and $w_{2}$ are the MRC weighting factors. According to the MRC criterion, the MRC weighting factors are configured as $w_{1}=\frac{h_{S 1}^{H}[n] \sqrt{P \alpha_{1}}}{\sigma^{2}}$, and $w_{2}=\frac{h_{R 1}^{H}[n+1] \rho h_{S R}^{H}[n] \sqrt{P \alpha_{1}}}{\left(\mid h_{R 1}\left[n+\left.1\right|^{2} \rho^{2}+1\right) \sigma^{2}\right.}$. where ()$^{H}$ denotes the conjugate transpose. Hence, the signal-noise-ratio (SNR) for decoding symbol 1 by $\mathrm{U} 1$ can be expressed as

$$
\gamma_{1}=\alpha_{1} \gamma_{S 1}+\frac{\alpha_{1} \gamma_{S R} \gamma_{R 1}}{\gamma_{S R}+\gamma_{R 1}+1}
$$

$\mathrm{U} 2$ receives the AF signal $y_{2}[n+1]$ by the relay. The SNR for decoding symbol 2 by U2 is given by

$$
\gamma_{2}=\frac{\alpha_{2} \gamma_{S R} \gamma_{R 2}}{\alpha_{1} \gamma_{S R} \gamma_{R 2}+\gamma_{S R}+\gamma_{R 2}+1}
$$

Hence, the ergodic capacity of our proposed system can be given by

$$
C_{\text {proposed }}=\frac{1}{2} E\left[\log _{2}\left(1+\alpha_{1} \gamma_{S 1}+\frac{\alpha_{1} \gamma_{S R} \gamma_{R 1}}{\gamma_{S R}+\gamma_{R 1}+1}\right)+\frac{1}{2} \log _{2}\left(1+\frac{\alpha_{2} \gamma_{S R} \gamma_{R 2}}{\alpha_{1} \gamma_{S R} \gamma_{R 2}+\gamma_{S R}+\gamma_{R 2}+1}\right)\right] .
$$

As a comparative analysis, in [16], to deal with $y_{1}[n]$ and $y_{1}[n+1]$ at the user U1, where first applies MRC next applies SIC to $y_{1}[n]$ and $y_{1}[n+1]$. Thus, the ergodic capacity of [16] proposed system can be written as

$$
\begin{aligned}
C_{[16]}= & \frac{1}{2} E\left[\log _{2}\left(1+\frac{\alpha_{1} \gamma_{S 1}^{2}\left(\gamma_{S R}+1\right)^{2}+\alpha_{1} \gamma_{S R} \gamma_{R 1}\left(\gamma_{S R} \gamma_{R 1}+2 \gamma_{S R} \gamma_{S 1}+2 \gamma_{S 1}\right)}{\gamma_{S 1}\left(\gamma_{S R}+1\right)^{2}+\gamma_{S R} \gamma_{R 1}\left(\gamma_{S R}+\gamma_{R 1}+1\right)}\right)\right. \\
& \left.+\log _{2}\left(1+\frac{\alpha_{2} \gamma_{S R} \gamma_{R 2}}{\alpha_{1} \gamma_{S R} \gamma_{R 2}+\gamma_{S R}+\gamma_{R 2}+1}\right)\right]
\end{aligned}
$$

\section{Ergodic Capacity Performance Analysis}

In this part, we study the ergodic capacity, which is an important performance metric of our proposed cooperative NOMA system. However, as the closed-form expression of the precise ergodic capacity for the system is hard to get. we provide an approximation of the closed-form expression for the ergodic capacity. The asymptotic characteristics of the ergodic capacity in the high SNR regime are also investigated.

In order to acquire the closed-form expression approximation of ergodic capacity for symbol 1 and symbol 2, the next four lemmas will be given first.

Lemma 1. Assuming the random variables $S$ and $T$ obey exponentially distributed and the mean are $\beta_{1}$ and $\beta_{2}$, respectively. If $x, y, z, w$, and $u$ are positive constant and $x-y r>0$, the cumulative distribution function (CDF) of $R=\frac{x S T}{y S T+z S+w T+u}$ is given by

$$
F_{R}(r)=1-\frac{e^{-\frac{r}{x-y r}\left(\frac{w}{\beta_{1}}+\frac{z}{\beta_{2}}\right)}}{\beta_{2}(x-y r)} \sqrt{\frac{4 \beta_{2}}{\beta_{1}}\left(w z r^{2}+u r(x-y r)\right)} K_{1}\left(\sqrt{\frac{4}{\beta_{1} \beta_{2}}\left(\frac{w z r^{2}}{(x-y r)^{2}}+\frac{u r}{x-y r}\right)}\right),
$$


where $K_{1}(x)$ represents the second kind modified bessel function.

Proof. According to the definition of random variable $R$, its CDF can be given by

$$
\begin{aligned}
F_{R}(r) & =P(R<r) \\
& =P\left(\frac{x S T}{y S T+z S+w T+u}<r\right) \\
& =P(S(x T-y r T-z r)<w T r+u r),
\end{aligned}
$$

If $x T-y r T-z r<0$, then

$$
\begin{aligned}
F_{R}(r) & =P(S(x T-y r T-z r)<w T r+u r) \\
& =P\left(S>\frac{w T r+u r}{x T-y r T-z r}\right) \\
& =1 .
\end{aligned}
$$

else

$$
\begin{aligned}
F_{R}(r) & =P(S(x T-y r T-z r)<w T r+u r) \\
& =P\left(S<\frac{w T r+u r}{x T-y r T-z r}\right) \\
& =F_{S}\left(\frac{w T r+u r}{x T-y r T-z r}\right) .
\end{aligned}
$$

From the discussion above, the CDF of the random variable $R$ can be further given by

$$
\begin{aligned}
F_{R}(r) & =\int_{0}^{\frac{z r}{x-y r}} f_{T}(t) d t+\int_{\frac{z r}{x-y r}}^{+\infty}\left(1-e^{-\frac{w t r+u r}{\beta_{1}(x t-y r t-z r)}}\right) f_{T}(t) d t \quad(q=x t-y r t-z r) \\
& =1-\frac{e^{-\frac{r}{x-y r}\left(\frac{w}{\beta_{1}}+\frac{z}{\beta_{2}}\right)}}{\beta_{2}(x-y r)} \int_{0}^{+\infty} e^{-\frac{1}{q}\left(\frac{\frac{w r^{2}}{x-y r}+u r}{\beta_{1}}\right)} e^{-q\left(\frac{1}{\beta_{2}(x-y r)}\right)} d q .
\end{aligned}
$$

Giving equation (3.324-1) in [17]

$$
\int_{0}^{\infty} \exp \left(-\frac{\beta}{4 x}-\gamma x\right) d x=\sqrt{\frac{\beta}{\gamma}} K_{1}(\sqrt{\beta \gamma}) \quad \operatorname{Re} \beta \geq 0, \operatorname{Re} \gamma \geq 0,
$$

By utilizing the equation (17), according to correspondence coefficient rule, the expression (12) can be acquired.

Lemma 2. Based on the assumptions for Lemma 1, furthermore, if $\beta_{1} \rightarrow+\infty$ and $\beta_{2} \rightarrow+\infty$, the $C D F$ of the random variable $R$ is given by

$$
F_{R}^{\infty}(r)=\frac{w r}{\beta_{1}(x-y r)}+\frac{z r}{\beta_{2}(x-y r)} .
$$

Proof. By utilizing the equation (9.7.2) in [18], we can have the following approximation

$$
K_{1}\left(\frac{1}{x}\right)=x, x \rightarrow \infty .
$$

Then, by using the approximation (19), we can simplify the expression (12) as

$$
F_{R}^{\infty}(r)=1-e^{-\frac{w r}{\beta_{1}(x-y r)}-\frac{z r}{\beta_{2}(x-y r)}} .
$$

Finally, by applying $\lim _{x \rightarrow 0} \frac{1-e^{-x}}{x}=1$, the equation (20) can be further expressed as (18) . 
Thus, Lemma 2 is proved.

Lemma 3. Assuming the random variables $X>0$, the probability density function (PDF) and the $C D F$ of $X$ can be expressed as $f_{X}(x)$ and $F_{X}(x)$, respectively. the following expression exists

$$
\int_{0}^{\infty} \log _{2}(1+x) f_{X}(x) d x=\frac{1}{\ln 2} \int_{0}^{\infty} \frac{1-F_{X}(x)}{1+x} d x .
$$

Proof. The proof process is as follows

$$
\begin{aligned}
\int_{0}^{\infty} \log _{2}(1+x) f_{X}(x) d x & =\int_{0}^{\infty} \log _{2}(1+x) d F_{X}(x) \\
& =\left.\log _{2}(1+x) F_{X}(x)\right|_{0} ^{\infty}-\int_{0}^{\infty} F_{X}(x) d \log _{2}(1+x) \\
& =\left.\log _{2}(1+x)\right|_{0} ^{\infty}-\int_{0}^{\infty} F_{X}(x) d \log _{2}(1+x) \\
& =\int_{0}^{\infty} d \log _{2}(1+x)-\int_{0}^{\infty} F_{X}(x) d \log _{2}(1+x) \\
& =\frac{1}{\ln 2} \int_{0}^{\infty} \frac{1-F_{X}(x)}{1+x} d x
\end{aligned}
$$

The proof is completed.

Lemma 4. Assuming the random variables $X$ obey exponentially distributed and the mean is $\lambda_{x}$. If $a$ and $b$ are positive constant, the CDF of $Y=\frac{a X}{b X+1}$ is given by

$$
F_{Y}(y)=1-e^{-\frac{y}{\lambda_{x}(a-b y)}}\left(\varepsilon(y)-\varepsilon\left(y-\frac{a}{b}\right)\right)
$$

where $\varepsilon(x)$ represents step function, $\varepsilon(x)=\left\{\begin{array}{ll}1, & x \geq 0 \\ 0, & \text { else }\end{array}\right.$.

Proof. According to the definition of random variable $Y$, its CDF can be expressed as

if $a-b y>0$, then

$$
\begin{aligned}
F_{Y}(y) & =P(Y<y) \\
& =P\left(\frac{a X}{b X+1}<y\right) \\
& =P(X(a-b y)<y),
\end{aligned}
$$

$$
\begin{aligned}
F_{Y}(y) & =P\left(X<\frac{y}{a-b y}\right) \\
& =F_{X}\left(\frac{y}{a-b y}\right) \\
& =1-e^{-\frac{y}{\lambda_{x}(a-b y)}},
\end{aligned}
$$

else 


$$
\begin{aligned}
F_{Y}(y) & =P\left(X>\frac{y}{a-b y}\right) \\
& =1 .
\end{aligned}
$$

According to (25) and (26), the (23) is proved.

Proposition 1. Based on high SNR regime, an upper bound approximation ergodic capacity for the symbol 1 of our proposed system is given by

$$
E^{h i g h}\left[R_{1}\right]=\frac{1}{2 \ln 2}\left[m e^{\frac{1}{\alpha_{1} \lambda_{S 1}}} \operatorname{Ei}\left(-\frac{1}{\alpha_{1} \lambda_{S 1}}\right)-(m+1) e^{\frac{\lambda_{S R}+\lambda_{R 1}}{\alpha_{1} \lambda_{S R} \lambda_{R 1}}} \operatorname{Ei}\left(-\frac{\lambda_{S R}+\lambda_{R 1}}{\alpha_{1} \lambda_{S R} \lambda_{R 1}}\right)\right],
$$

where $m=\frac{\lambda_{S 1}\left(\lambda_{R 1}+\lambda_{S R}\right)}{\lambda_{S R} \lambda_{R 1}-\lambda_{S 1}\left(\lambda_{R 1}+\lambda_{S R}\right)}, \operatorname{Ei}(x)=\int_{-x}^{\infty} \frac{e^{-t}}{t} d t . E i(x)$ is exponential integral.

Proof. The ergodic capacity of the symbol 1 is expressed as

$$
\begin{aligned}
E\left[R_{1}\right] & =E\left[\frac{1}{2} \log _{2}\left(1+\alpha_{1} \gamma_{S 1}+\frac{\alpha_{1} \gamma_{S R} \gamma_{R 1}}{\gamma_{S R}+\gamma_{R 1}+1}\right)\right] \\
& =E\left[\frac{1}{2} \log _{2}\left(1+\alpha_{1} \gamma_{S 1}+\frac{\alpha_{1} \gamma_{S R} \gamma_{R 1}}{\gamma_{S R}+\gamma_{R 1}+1}\right)\right] \\
& =E\left[\frac{1}{2} \log _{2}(1+R)\right] \\
& =\int_{0}^{+\infty} \frac{1}{2} \log _{2}(1+R) f_{R}(r) d r
\end{aligned}
$$

we assumed that $S=\alpha_{1} \gamma_{S 1}$ and $T=\frac{\alpha_{1} \gamma_{S R} \gamma_{R 1}}{\gamma_{S R}+\gamma_{R 1}+1}$, hence, existing $f_{S}(s)=\frac{1}{\alpha_{1} \lambda_{S 1}} e^{-\frac{s}{\alpha_{1} \lambda_{S 1}}}$. By utilizing the (19) for the random variable $T$, we can obtain that $F_{T}(t) \geq 1-e^{-\frac{t\left(\lambda_{S R}+\lambda_{R 1}\right)}{\alpha_{1} \lambda_{S R} \lambda_{R 1}}}$, thus

$$
\begin{aligned}
F_{R}(r) & =P(S+T<r) \\
& =P(T<r-S) \\
& \geq \int_{0}^{r}\left(1-e^{\frac{s-r}{\alpha_{1} \lambda_{s r}}+\frac{s-r}{\alpha_{1} \lambda_{r 1}}}\right) \frac{1}{\alpha_{1} \lambda_{s 1}} e^{-\frac{s}{\alpha_{1} \lambda_{s 1}}} d s,
\end{aligned}
$$

The derivative of random variable $r$ on both sides of the inequality becomes

$$
f_{R}(r) \leq-m \frac{1}{\alpha_{1} \lambda_{S 1}} e^{-\frac{r}{\alpha_{1} \lambda_{S 1}}}+(m+1) \frac{\lambda_{R 1}+\lambda_{S R}}{\alpha_{1} \lambda_{S R} \lambda_{R 1}} e^{-\frac{\lambda_{R 1}+\lambda_{S R}}{\alpha_{1} \lambda_{S R} \lambda_{R 1}}},
$$

where $m=\frac{\lambda_{S 1}\left(\lambda_{R 1}+\lambda_{S R}\right)}{\lambda_{S R} \lambda_{R 1}-\lambda_{S 1}\left(\lambda_{R 1}+\lambda_{S R}\right)}$, next, giving equation (4.337-2) in [18]

$$
\int_{0}^{\infty} e^{-\mu x} \ln (1+\beta x) d x=-\frac{1}{\mu} e^{\frac{\mu}{\beta}} \operatorname{Ei}\left(-\frac{\mu}{\beta}\right) \quad|\arg \beta|<\pi, \operatorname{Re} \mu>0 .
$$

By utilizing the expression (31), according to correspondence coefficient rule, the expression (27) can be obtained.

Proposition 2. Based on high SNR regime, an upper bound approximation ergodic capacity for the symbol 2 of the proposed system is given by 


$$
E^{\infty}\left[R_{2}\right]=\frac{1}{2} \log _{2}\left(1+\frac{\alpha_{2}}{\alpha_{1}}\right) .
$$

Proof. The ergodic capacity of the symbol 2 is expressed as

$$
E\left[R_{2}\right]=\frac{1}{2} \log _{2}\left(1+\frac{\alpha_{2} \gamma_{S R} \gamma_{R 2}}{\alpha_{1} \gamma_{S R} \gamma_{R 2}+\gamma_{S R}+\gamma_{R 2}+1}\right),
$$

when the symbol 2 in high SNR, equivalent to $\gamma_{S R} \rightarrow \infty$ and $\gamma_{R 2} \rightarrow \infty$, the expression (32) can be obtained.

Proposition 3. Based on high SNR regime, an upper bound approximation ergodic capacity for the proposed system is given by

$$
E^{\infty}\left[R_{\text {sum }}\right]=\frac{1}{2} \log _{2}\left(\lambda_{S 1}\right)+\frac{\lambda_{S R} \lambda_{R 1}}{2\left[\lambda_{S R} \lambda_{R 1}-\lambda_{S 1}\left(\lambda_{S R}+\lambda_{R 1}\right)\right]} \log _{2}\left(\frac{\lambda_{S R} \lambda_{R 1}}{\lambda_{S 1}\left(\lambda_{S R}+\lambda_{R 1}\right)}\right)-\frac{E c}{2 \ln 2},
$$

where $E c \approx 0.5772$.

Proof. If $x \rightarrow 0$, existing $e^{x}=1+x$ and $E i(-x)=\ln x+E c$. Based on the symbol 1 in high SNR, equivalent to $\gamma_{S R} \rightarrow \infty, \gamma_{R 1} \rightarrow \infty$ and $\gamma_{S 1} \rightarrow \infty$, thus, $E^{\text {high }}\left[R_{1}\right]$ is given by

$$
\begin{aligned}
E^{\text {high }}\left[R_{1}\right] & =\frac{1}{2 \ln 2}\left[m e^{\frac{1}{\alpha_{1} \lambda_{S 1}}} \operatorname{Ei}\left(-\frac{1}{\alpha_{1} \lambda_{S 1}}\right)-(m+1) e^{\frac{\lambda_{S R}+\lambda_{R 1}}{\alpha_{S R} \lambda_{R 1}}} \operatorname{Ei}\left(-\frac{\lambda_{S R}+\lambda_{R 1}}{\alpha_{1} \lambda_{S R} \lambda_{R 1}}\right)\right] \\
& \leq \frac{1}{2 \ln 2}\left[m E i\left(-\frac{1}{\alpha_{1} \lambda_{S 1}}\right)-(m+1) E i\left(-\frac{\lambda_{S R}+\lambda_{R 1}}{\alpha_{1} \lambda_{S R} \lambda_{R 1}}\right)\right] \\
& =\frac{1}{2 \ln 2}\left[m\left(-\ln \alpha_{1} \lambda_{S 1}+E c\right)-(m+1)\left(\ln \frac{\lambda_{S R}+\lambda_{R 1}}{\alpha_{1} \lambda_{S R} \lambda_{R 1}}+E C\right)\right] \\
& =\frac{1}{2} \log \alpha_{1} \lambda_{S 1}+\frac{\lambda_{S R} \lambda_{R 1}}{2\left[\lambda_{S R} \lambda_{R 1}-\lambda_{S 1}\left(\lambda_{S R}+\lambda_{R 1}\right)\right]} \log _{2}\left(\frac{\lambda_{S R} \lambda_{R 1}}{\lambda_{S 1}\left(\lambda_{S R}+\lambda_{R 1}\right)}\right)-\frac{E C}{2 \ln 2} \\
& =E^{\infty}\left[R_{1}\right],
\end{aligned}
$$

by performing addition operation on the (32) and (35), the proof is completed.

Based on the high SNR regime, some important intuitive conclusions are as follows, on the one hand, the ergodic capacity of symbol 2 for our proposed system is only determined by the power allocation coefficient ratio, namely, the ergodic capacity of symbol 2 increases as the power allocation coefficient $\alpha_{2}$ increases. On the other hand, we also can see that, the power allocation coefficient has little effect on the ergodic capacity of our proposed system. From the above two conclusions, we can infer that the ergodic rate of symbol 1 increases as the power allocation coefficient $\alpha_{1}$ increases.

In order to effectively evaluate the ergodic capacity of performance between our proposed system and other two conventional cooperative NOMA systems. Here, the ergodic capacity of conventional cooperative NOMA system with DF relay and the conventional cooperative NOMA system with AF relay are respectively given by

$$
\begin{gathered}
C_{\text {con_DF }}=\frac{1}{2} E\left[\log _{2}\left(1+\alpha_{1} \gamma_{S 1}\right)+\frac{1}{2} \log _{2}\left(1+\min \left\{\frac{\alpha_{2} \gamma_{S R}}{\alpha_{1} \gamma_{S R}+1}, \gamma_{R 2}\right\}\right)\right], \\
C_{c n_{-} A F}=\frac{1}{2} E\left[\log _{2}\left(1+\alpha_{1} \gamma_{S 1}\right)+\frac{1}{2} \log _{2}\left(1+\frac{\alpha_{2} \gamma_{S R} \gamma_{R 2}}{\alpha_{1} \gamma_{S R} \gamma_{R 2}+\gamma_{S R}+\gamma_{R 2}+1}\right)\right] .
\end{gathered}
$$


In two conventional cooperative NOMA systems, symbol 1 has no chance to do MRC at the second time slot. Thus, the conventional cooperative NOMA systems can't obtain diversity gain.

Next, two propositions is also given to describe the ergodic capacity analysis expression for two symbols of the two conventional cooperative NOMA systems.

Proposition 4. the ergodic capacity for the symbol 1 of the conventional cooperative NOMA system with DF/AF relay is given by

$$
E_{\text {con }}\left[R_{1}\right]=-\frac{1}{2 \ln 2} e^{\frac{1}{\alpha_{1} \lambda_{s 1}}} \operatorname{Ei}\left(-\frac{1}{\alpha_{1} \lambda_{s 1}}\right),
$$

Proof. The ergodic rate of the symbol 1 for the conventional cooperative NOMA system with $\mathrm{DF} / \mathrm{AF}$ relay is expressed as

$$
E_{\text {con }}\left[R_{1}\right]=\frac{1}{2} E\left[\log _{2}\left(1+\alpha_{1} \gamma_{s 1}\right)\right],
$$

where $\gamma_{S 1}$ obey exponential distributed and its means is $\lambda_{S 1}$, and we define that $X=\alpha_{1} \gamma_{S 1}$. The CDF of $X$ is given by

$$
F_{X}(x)=1-e^{-\frac{x}{\alpha_{1} \lambda_{s 1}}}
$$

By utilizing Lemma 3, the $E\left[R_{1}\right]$ can be further expressed as

$$
E_{\text {con }}\left[R_{1}\right]=\frac{1}{2 \ln 2} \int_{0}^{\infty} \frac{e^{-\frac{x}{\alpha_{1} \lambda_{s 1}}}}{1+x} d x .
$$

Giving equation (3.352-4) in [18]

$$
\int_{0}^{\infty} \frac{e^{-\mu x}}{x+\beta} d x=-e^{\beta \mu} \operatorname{Ei}(-\mu \beta) \quad|\arg \beta|<\pi, \operatorname{Re} \mu>0 .
$$

By utilizing the equation (42) and (41), according to correspondence coefficient rule, the expression (38) can be obtained, Proposition 4 is proved.

Proposition 5. the ergodic capacity for the symbol 2 of the conventional cooperative NOMA system with DF relay is given by

$$
E_{\text {con }}\left[R_{2}\right]=\frac{1}{2 \ln 2} \int_{0}^{\frac{\alpha_{2}}{\alpha_{1}}} e^{-x\left(\frac{1}{\lambda_{\text {SR }}\left(a_{2}-a_{1} x\right)}+\frac{1}{\lambda_{R 2}}\right)} \frac{1}{1+x} d x .
$$

Proof. According to (36), we define that $Z=\min \left\{\frac{\alpha_{2} \gamma_{S R}}{\alpha_{1} \gamma_{S R}+1}, \gamma_{R 2}\right\}$, the CDF of the variable $Z$ is given by

$$
\begin{aligned}
F_{Z}(x) & =P\left(\min \left\{\frac{\alpha_{2} \gamma_{S R}}{\alpha_{1} \gamma_{S R}+1}, \gamma_{R 2}\right\}<x\right) \\
& =1-P\left(\frac{\alpha_{2} \gamma_{S R}}{\alpha_{1} \gamma_{S R}+1} \geq x\right) P\left(\gamma_{R 2} \geq x\right),
\end{aligned}
$$

By utilizing Lemma 4, the CDF of the variable $Z$ can be further expressed as

$$
\begin{aligned}
F_{Z}(x) & =1-P\left(\frac{\alpha_{2} \gamma_{S R}}{\alpha_{1} \gamma_{S R}+1} \geq x\right) P\left(\gamma_{R 2} \geq x\right) \\
& =1-e^{-\frac{x}{\lambda_{S R}\left(a_{2}-a_{1} x\right)}} e^{-\frac{x}{\lambda_{R 2}}}\left(\varepsilon(x)-\varepsilon\left(x-\frac{a_{2}}{a_{1}}\right)\right) .
\end{aligned}
$$


Bring (45) to Lemma 3, the proof is completed.

The best knowledge of the authors, based on the conventional cooperative NOMA system with DF relay, the closed-form expression of ergodic capacity for symbol 2 was not obtained. However, the ergodic rate also can be effectively estimated via the definite integration (43). In addition, based on the conventional cooperative NOMA system with AF relay, we do not give the proposition to describe the analysis expression of the ergodic rate for symbol 2 . This main because that the ergodic rate is identical for symbol 2 between our proposed system and the conventional cooperative NOMA system with AF relay. The proposition 2 has given an upper bound approximation for ergodic capacity based on symbol 2.

\section{Numerical Results}

In this section, the ergodic capacity of all cooperative NOMA systems are evaluated by Monte-Carlo simulations, based on 10,000 independent random channel realizations. For all simulations, we set $\lambda_{S R}=14, \lambda_{S 1}=5, \lambda_{R 1}=10, \lambda_{R 2}=10, \sigma^{2}=1$.

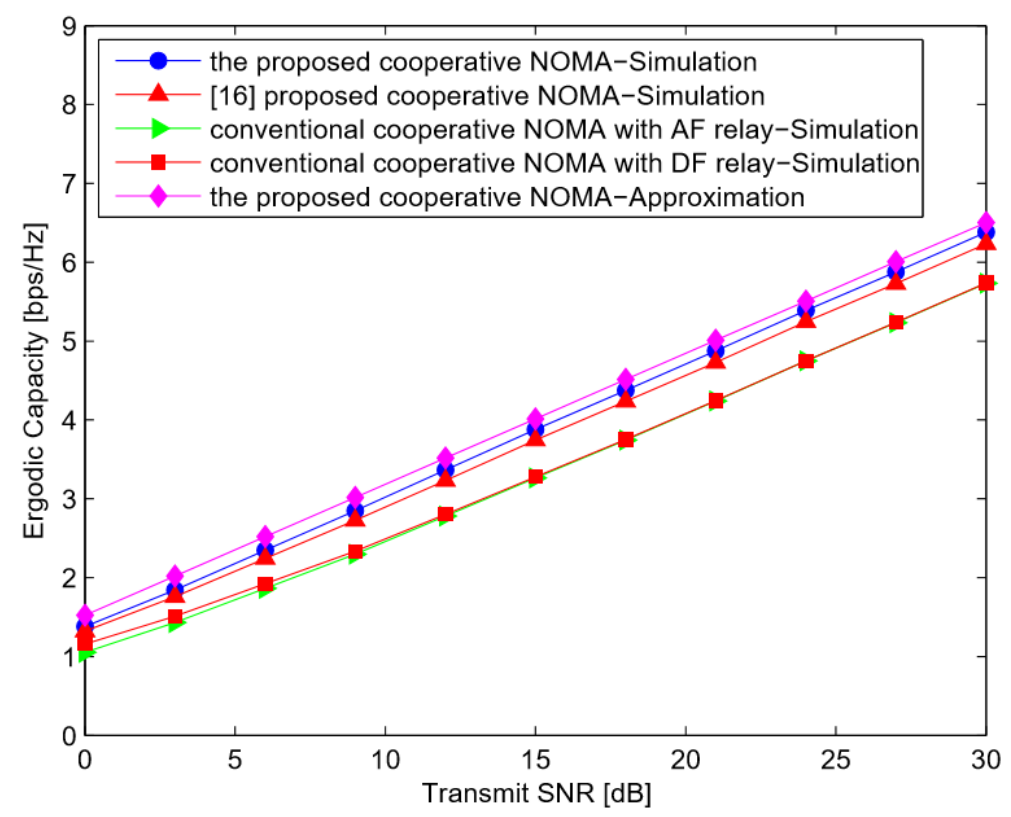

Fig. 2. Ergodic capacity versus transmit SNR for all compared cooperative NOMA systems

In Fig. 2, we compare the ergodic capacity among our proposed system, conventional cooperative NOMA systems with AF/DF relay and [16] proposed cooperative NOMA system. In simulations, we further set power allocation coefficients $\alpha_{1}=0.4, \alpha_{2}=0.6$. As we can see from this figure, our proposed system has better ergodic capacity than the other three systems. This is due to two reasons. First of all, our proposed cooperative NOMA system utilizes MRC for symbol 1 , so the symbol 1 obtain two signals ergodic capacity gain from two time slots. However, two conventional cooperative NOMA systems can't obtain diversity gain for symbol 1 due to not applying MRC at U1. On the other hand, at the second time slot, comparing the strategy in [16] of performing MRC before SIC, our detection scheme for symbol 1 can obtain better diversity gain. Hence, our proposed system achieves the best ergodic capacity performance. In addition, we also see that ergodic capacity increases with increasing transmit 
SNR for all compared system. Besides, we see approximated ergodic capacity of our proposed system given by (34) which is an upper bound for simulation ergodic capacity.

Fig. 3 presents the ergodic capacity versus power allocation coefficient for our proposed system, two conventional cooperative NOMA systems and [16] proposed cooperative NOMA system. As can be seen from the figure, under fixed transmit SNR, approximated ergodic capacity upper bound of our proposed system is changeless with the increase of power allocation coefficient. This is due to that approximated ergodic capacity upper bound is only determined by transmit SNR and channel power gains, as expression (34). Also, ergodic capacity of all compared systems are improved by increasing transmit SNR. In addition, from this figure, we also find that power allocation coefficients have little impact on ergodic capacity of all cooperative NOMA systems.

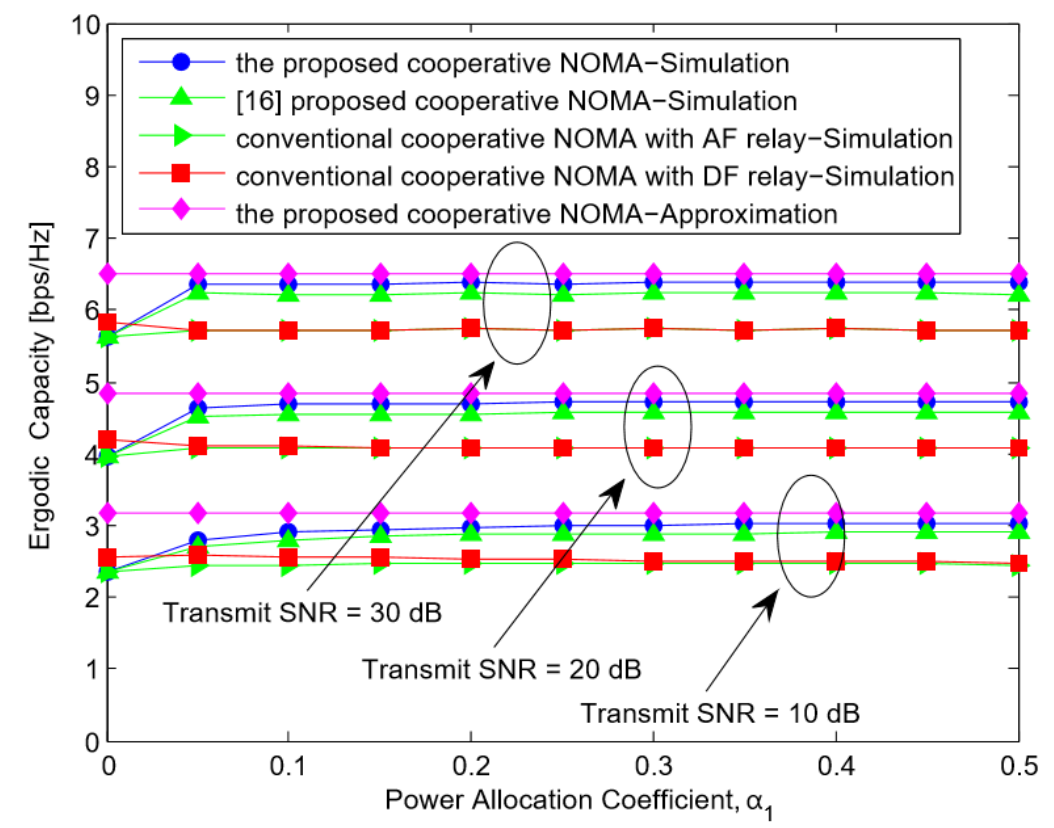

Fig. 3. Ergodic capacity versus power allocation coefficient for all compared cooperative NOMA systems

Fig. 4 depicts the ergodic capacity versus transmit SNR and power allocation coefficient 1 for our proposed cooperative NOMA system, using Monte-Carlo simulations. From this figure we can see that ergodic capacity of our proposed system significantly increases with the increase of transmit SNR. However, we also see that power allocation coefficients have little impact on ergodic capacity of our proposed cooperative NOMA system. The corresponding reason will be explained in the analysis part of Fig. 5 .

Fig. 5 shows the ergodic capacity versus power allocation coefficient 1 for our proposed cooperative NOMA system. In simulations, we further set transmit SNR $\rho=30 \mathrm{~dB}$. From the figure we can see that the ergodic capacity of each symbol increases with the increase of its own power allocation coefficient, in which satisfy $\alpha_{2}>\alpha_{1}$ and $\alpha_{1}+\alpha_{2}=1$. In other words, the ergodic capacity of symbol 1 increases with the increase of power allocation coefficient 1 . However, the ergodic capacity of symbol 2 decreases with the increase of power allocation coefficient 1 . Hence, we can understand that power allocation coefficients have little impact on ergodic capacity of our proposed cooperative NOMA system and the other three 
cooperative NOMA systems. This is because the ergodic capacity of two symbols can be complementary with changing of power allocation coefficients.

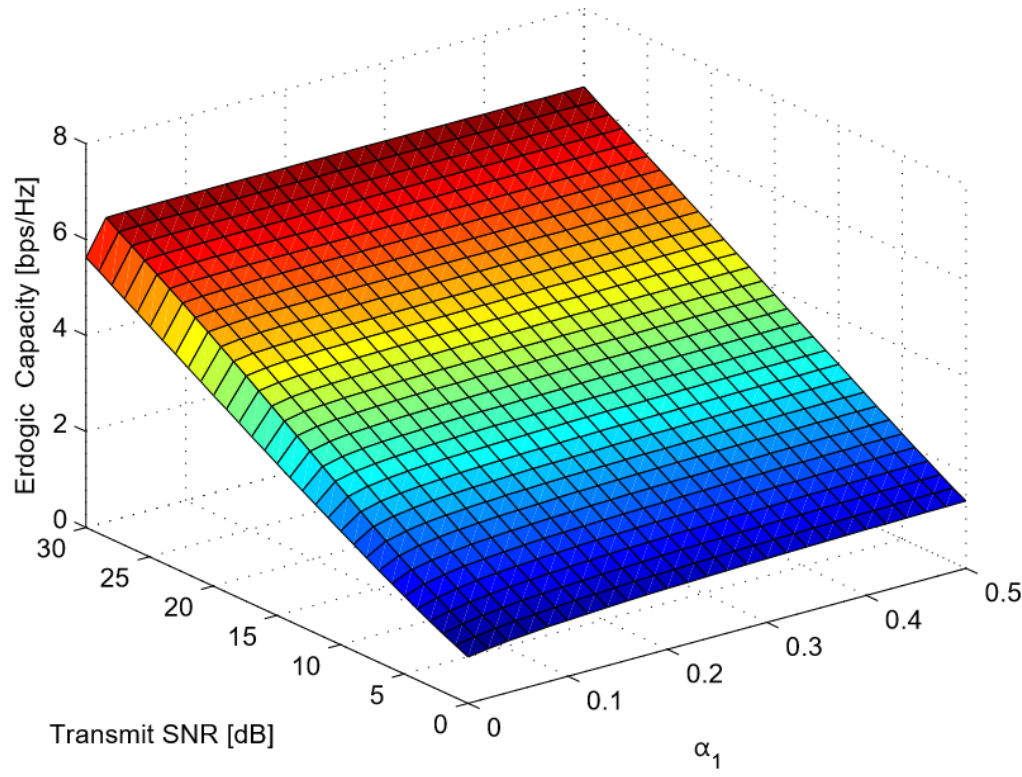

Fig. 4. Ergodic capacity versus power allocation coefficient and transmit SNR for the proposed cooperative NOMA system

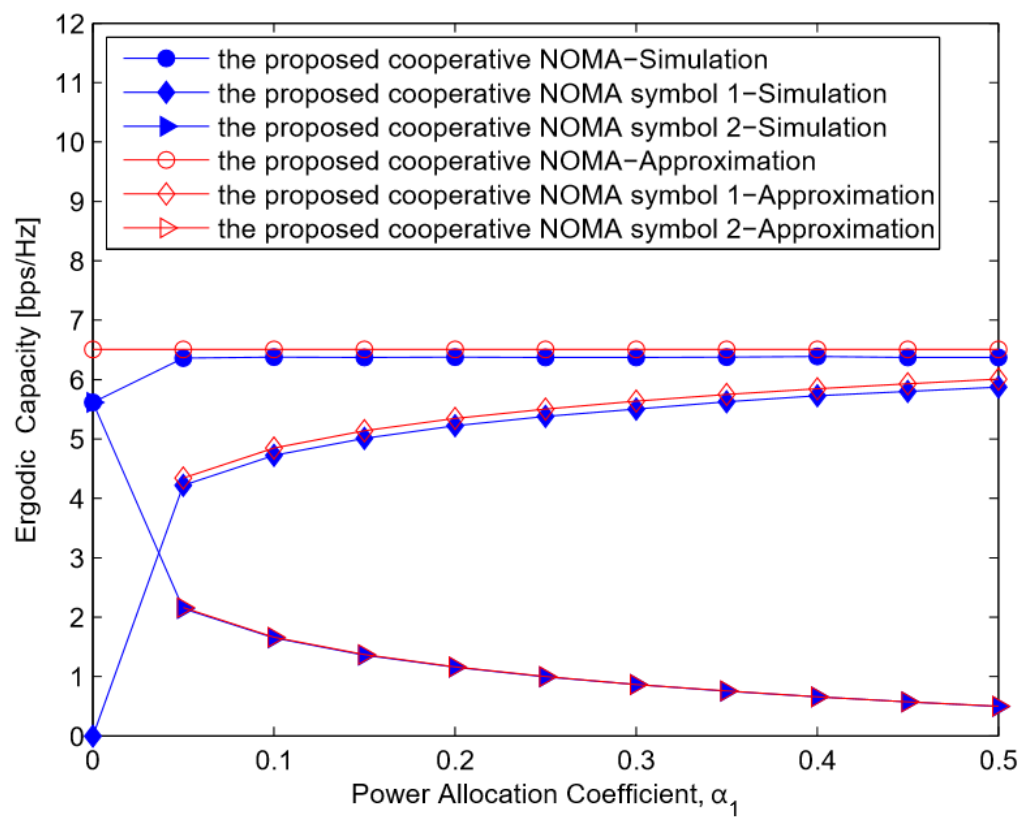

Fig. 5. Ergodic capacity versus power allocation coefficient for the proposed cooperative NOMA system 


\section{Conclusion}

This paper proposes an improved NOMA system with the help of an AF relay. A closed-form upper bound approximation is derived for the ergodic capacity of our proposed system. A novel detection scheme is applied at U1. Monte-Carlo simulations and numerical analysis show that our proposed system can obtains higher ergodic capacity than the conventional cooperative NOMA systems with DF/AF relay system and [16] proposed cooperative NOMA system. In addition, the ergodic capacity of our proposed system increases as transmit SNR increases. Finally, simulations also indicate that power allocation coefficients have little impact on ergodic capacity of all cooperative NOMA systems. This is because ergodic capacity of two symbols can be complementary with changing of power allocation coefficients.

\section{Acknowledgement}

This work has been supported by the Joint Foundation of Guizhou Province (QianKeHeLHZi[2016]7106), XJGC20150702 and the Nature Science Foundation of Guizhou Province (QianKeHeJiChu[2017]1070).

\section{References}

[1] L. Dai, B. Wang, Y. Yuan, S. Han, I. Chih-Lin, and Z. Wang, "Non-orthogonal multiple access for 5G: solutions, challenges, opportunities, and future research trends," IEEE Communications Magazine, vol. 53, no. 9, pp. 74-81, September, 2015. Article (CrossRef Link).

[2] Y. Chen, A. Bayesteh, Y. Wu, B. Ren, S. Kang, S. Sun, Q. Xiong, C. Qian, B. Yu, Z. Ding, S. Wang, S. Han, X. Hou, H. Lin, R. Visoz, and R. Razavi, "Toward the standardization of non-orthogonal multiple access for next generation wireless networks," IEEE Communications Magazine, vol. 56, no. 3, pp. 19-27, March, 2018. Article (CrossRef Link).

[3] G. Gui, H. Sari, and E. Biglieri, "A new definition of fairness for non-orthogonal multiple access,” IEEE Communications Letters, vol. 23, no. 7, pp. 1267-1271, July, 2019. Article (CrossRef Link).

[4] J. Wang, M. Liu, J. Sun, G. Gui, H. Gacanin, H. Sari, and F. Adachi, "Multiple unmanned aerial vehicles deployment and user pairing for non-orthogonal multiple access schemes," IEEE Internet Things of Journal, vol. 14, no. 8, pp. 1-13, August, 2020. Article (CrossRef Link).

[5] G. Gui, M. Liu, F. Tang, N. Kato, and F. Adachi, "6G: Opening new horizons for integration of comfort, security and intelligence,” IEEE Wireless Communications, pp.1-7, 2020. Article (CrossRef Link).

[6] Z. Ding, X. Lei, G. K. Karagiannidis, R. Schober, J. Yuan, and V. K. Bhargava, “A survey on non-orthogonal multiple access for 5G networks: Research challenges and future trends," IEEE Journal on Selected Areas in Communications, vol. 35, no. 10, pp. 2181-2195, October, 2017. Article (CrossRef Link).

[7] M. Xu, F. Ji, M. Wen, and W. Duan, "Novel receiver design for the cooperative relaying system with non-orthogonal multiple access," IEEE Communications Letters, vol. 20, no. 8, pp. 1679-1682, August, 2016. Article (CrossRef Link).

[8] Z. Ding, H. Dai, and H. V. Poor, "Relay selection for cooperative NOMA," IEEE Wireless Communications Letters, vol. 5, no. 4, pp. 416-419, August, 2016. Article (CrossRef Link).

[9] M. S. Elbamby, M. Bennis, W. Saad, M. Debbah, and M. Latva-aho, "Resource optimization and power allocation in in-band full duplex-enabled non-orthogonal multiple access networks," IEEE Journal on Selected Areas in Communications, vol. 35, no. 12, pp. 2860-2873, December, 2017. Article (CrossRef Link). 
[10] C. Zhong and Z. Zhang, "Non-orthogonal multiple access with cooperative full-duplex relaying," IEEE Communications Letters, vol. 20, no. 12, pp. 2478-2481, December, 2016. Article (CrossRef Link).

[11] X. Yue, Y. Liu, S. Kang, A. Nallanathan, and Z. Ding, "Exploiting full/half-duplex user relaying in NOMA systems,” IEEE Transactions on Communications, vol. 66, no. 2, pp. 560-575, February, 2018. Article (CrossRef Link).

[12] J. Men and J. Ge, “Non-orthogonal multiple access for multiple-antenna relaying networks,” IEEE Communications Letters, vol. 19, no. 10, pp. 1686-1689, October, 2015. Article (CrossRef Link).

[13] X. Yue, Y. Liu, S. Kang, A. Nallanathan, and Y. Chen, "Modeling and analysis of two-way relay non-orthogonal multiple access systems," IEEE Transactions on Communications, vol. 66, no. 9, pp. 3784-3796, September, 2018. Article (CrossRef Link).

[14] W. Shin, H. Yang, M. Vaezi, J. Lee, and H. V. Poor, "Relay-aided NOMA in uplink cellular networks,” IEEE Signal Process Letter, vol. 24, no. 12, pp. 1842-1846, December, 2017. Article (CrossRef Link).

[15] Y. Liu, C. Gao, Y. Li, L. Su, and D. Jin, "Exploiting multi-hop relaying to overcome blockage in directional mmwave small cells," Journal of Communications and networks, vol. 18, no. 3, pp. 364-374, June, 2016. Article (CrossRef Link).

[16] X. Liang, Y. Wu, D. W. K. Ng, Y. Zuo, S. Jin, and H. Zhu, “Outage performance for cooperative NOMA transmission with an AF relay," IEEE Communications Letters, vol. 21, no. 11, pp. 2428-2431, November, 2017. Article (CrossRef Link).

[17] M. Abramowitz and I. A. Stegun, Handbook of mathematical functions: with formulas, graphs, and mathematical tables, Courier Corporation, 1964.

[18] I. S. Gradshteyn and I. M. Ryzhik, Table of integrals, series, and products, Academic press, 2014.

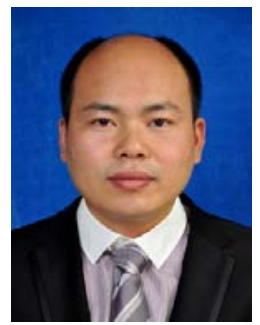

Xianbin Xie received the B.S. and M.S. degrees in Electronics and Communication Engineering form Hubei University of Technology, Wuhan, China, and the Ph.D. degree in Communication Engineering from UESTC, Chengdu, China, in 2003, 2008, and 2013, respectively. He has worked in the 38th Research Institute of China Electronics Technology Group since from 2013 to 2014 in Hefei, China. Since 2015, He has been with the Guizhou Institute of Technology, Guiyang, China, where he is currently a professor of the Department of Big Data Engineering. His research interests include cooperative communications, non-orthogonal multiple access, full-duplex and cognitive radio networks.

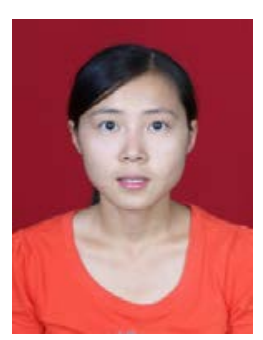

Yan Bi received the B.S. and M.S. degrees in Applied Mathematics from Xihua University ,Chengdu, China, in 2006 and 2014, respectively. She is working as an associate professor with the department of Mathematics and Physics, Guizhou Institute of Technology, China, and She is also currently pursuing the Ph.D. degree with the School of Mathematics and Statistics, Guizhou University, China. Her research interests include statistical signal analysis and processing.

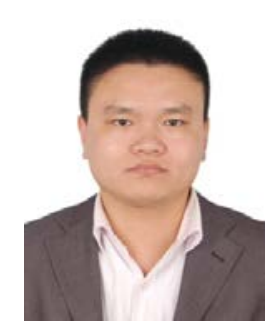

Xi Nie received the B.S and M.S degrees in electronics and communication engineering from Guizhou University, Guiyang, China, and the Ph.D. degree in communication engineering from UESTC, Chengdu, China, in 2003,2008, and 2015, respectively. He is currently working as an associate professor with the Department of Big Data Engineering, Guizhou Institute of Technology, China. His research interests focus on array signal processing. 